\title{
LED to LED communication with WDM concept for flash light of Mobile phones
}

\author{
Visible Light Communication
}

\author{
Devendra J Varanva \\ PG Student: ECE Department, \\ School of Engineering, RK University, \\ Rajkot, India
}

\author{
Kantipudi MVV Prasad \\ Assi. Prof.: ECE Department, \\ School of Engineering, RK University, \\ Rajkot, India
}

\begin{abstract}
Free Space Optical Communication especially Visible Light Communication, It is clear that LED is main component as a source. LED being solid state device makes endless list of possibilities. But here we will get through its ability to sense light as well, and use of Wavelength Division Multiplexing (WDM) in mobile flash is also suggested, this opens door to many applications.
\end{abstract}

Keywords-Free Space Light Communication; Visible Light Communicatio; Solid State Device; LED as Sensor; LED-LED Communication; WDM; VLC with Mobile handset/Smartphone.

\section{INTRODUCTION}

Free Space Optical Communication is emerging technology. It works in similar way like optical fiber network works but here air/free space shall be used as transmission medium. Today in different systems Laser, Infrared, and Ultraviolet Light used for communication over the air or in free space. This is actually needed at some places where wired connection is not easier or it is temporary connection. Like some years back use of IR communication in mobile handsets to share files was very popular until Bluetooth came. It has low speed but it was enough if we talk about that time. Best part of it was wireless connection which is better than connecting two phones with wire for such a short time of use. Being wireless has been dream of every human being. But here we are talking about free space communication using visible LEDs; popularly known as Visible Light Communication (Visible light is a part of electromagnetic spectrum which is perceivable by humans). People are talking much about it and they should, as this technology has very high potential to dominate communication market which is dominated by Radio Frequencies now.

Visible Light Communication is not a new technology. Since ancient times humans are using it in simple form. For example, in old times to give war signals, they used reflection of sunlight using brushed iron piece or smoke in day time and at night they were using fire to give signals. That was the best and very effective way to inform others by giving signal like this for that time as technology was not developed enough.

Today LED is very power efficient unlike fluorescent lights, tungsten bulb, etc. It is solid state device which can switch at very high speed that human eyes can't perceive. This property is matter of interest. We can send data with OOK (On-Off Keying) [1], OFDM [2], etc modulation schemes. To send data via Visible light communication we should switch
LED On and Off. When LED switched On, receiver will detect as " 1 " and when it switched Off, receiver should detect as "0".It is not as simple as sending and detecting, you need to use amplifier/filter at receiver end. Best part of Visible Light Communication is you can get advantage of high speed data communication at the same time you can use it for lighting purpose. By using this technology we can save lot of energy wasted in using $\mathrm{Wi}-\mathrm{Fi}$, as radio frequency can be used for only data communication purpose not lighting.

As we all know LED can be used for sensing purpose too. Publish by Forrest W. Mims [3] [4] in late 1970's but we have forgotten that. LED is nothing but PN diode. Working of Photodiode/sensor is quite similar. In our experiments we decided to use sensing capability of LEDs, Especially clear lens LED used in our experiments which gives best results.

\section{Potential Advantages of VLC}

Visible Light Communication has very unique advantages (especially over RF); some of them are explained here.

\section{A. Two functions : Lighting \& Data}

We use visible color LEDs for lighting purpose (as it supposed to be) as well as for Data Communication .Combining illumination and Communication is dual advantage for us unlike Radio Frequencies.

\section{B. Security}

If you are under the light/receiving the light means only you are receiving the data. e.g., one can use their light as hotspot to transmit data and you can receive data only where light reaches. So someone outside the room can't receive it if light is not going outside unlike Radio Frequencies where you cannot do same.

\section{It can work in hazardus zones}

We know that Radio Frequencies are not allowed in hazardous zone like nuclear plants, petroleum refineries, Hospitals, etc. But light used everywhere so we can install VLC hotspot for communication purpose, or to send current location data for automatic robot or other kind of machine and also can be used to send warning signal.

\section{Parallel Communication is also possible}

Researchers have already shown wavelength division multiplexing (WDM) capabilities for VLC. We can send 
different data on different line at the same time using different wavelength of the light [5].

\section{Challanges}

Advantages come at a cost in VLC, as it has some challenges to be resolved. (1)VLC is Line of Sight communication require you to align transmitter and receiver in straight line of sight. Without it VLC won't work. (2) Communication at long distances can't be possible due to dust or foggy environment, or in rain. In simple terms we can say if you can see the light you can receive the data. Despite many challenges, VLC is still very effective technology for future.

\section{LED AS A SENSOR}

Many researchers are working on this technology. It has been since Forrest M. Mims have shown that LED can act like a sensor to. This is the fact that clear lens LED can sense light with lower wavelengths than it emits. In short LED's sensitivity region is slightly wider then its spectral emission profile. So it can be used as a low cost option for some simple applications in place of LDR(Light Dependent Resistor) or Photodiodes(PD can detect wide spectrum of light).as LEDs are widely available at low cost. The fact is LEDs are intended to work for lighting purpose but we are using it in VLC for data communication too. And right now we are talking about using it as a photo sensor. But matter of interest is its capabilities to be used as a low speed photodiode which can have speed not more than some KBs. here we want to mention that LEDs can be used for bi-directional communication too using microcontroller by changing its polarity with two pins (both pins of LED connected to two I/O pins) [6] in which LED will show us property like capacitor, we first charge it and after changing its polarity we will calculate time to discharge. Here when you through light on it, will make it to discharge faster. We can calculate intensity of light by this way [7].

As shown in Table I. different color clear lens LED showed us different properties. We took Voltage change in LED when light is thrown to it. As we noted earlier LED can sense only wavelength emitted by it or from slightly near spectrum. Different Color LEDs have different characteristics as we can see in the table. Where green LED (Tx) to Orange LED (Rx) showed us best performance, also Blue LED (Tx) to Green LED (Rx).Specially for bi-directional communication (same LED for transmitter and receiver), Red LED might be good choice. As it can receive same light better emitted by its self.

Here the biggest advantage over Photodiode is, we don't need glass of other king of filter for filtering particular color of light, LED will do by itself. We have connected Anode to voltmeter and cathode to ground to measure voltage (displayed in Table. I). Resistor was used to control current of LED. It should be noted that these are the values for sensing purpose only we have to look at minor photocurrent generated by it and the frequency response of LED as sensor too. Different Color LEDs have different characteristics.
TABLE I. DIFFERENT COLOR LEDS AS PHOTO SENSOR

\begin{tabular}{|c|c|c|c|c|c|c|}
\hline \multirow{2}{*}{ LED } & \multicolumn{6}{|c|}{ Detector } \\
\hline & COLOR & $W$ & $\boldsymbol{R}$ & $G$ & $O$ & $B$ \\
\hline \multirow{5}{*}{$\begin{array}{c}\mathbf{E} \\
\mathbf{m} \\
\mathbf{i} \\
\mathbf{t} \\
\mathbf{t} \\
\mathbf{e} \\
\mathbf{r}\end{array}$} & WHITE & 11 & 430 & 405 & 420 & 30 \\
\hline & RED & 0 & 750 & 0 & 30 & 0 \\
\hline & GREEN & 0 & 450 & 105 & 1380 & 0 \\
\hline & ORANGE & 0 & 140 & 0 & 50 & 0 \\
\hline & BLUE & 54 & 30 & 1250 & 165 & 270 \\
\hline
\end{tabular}

b. performed in normal room light condition

distance between $\mathrm{Tx}$ and $\mathrm{Rx}$ was $7.5 \mathrm{~cm}$

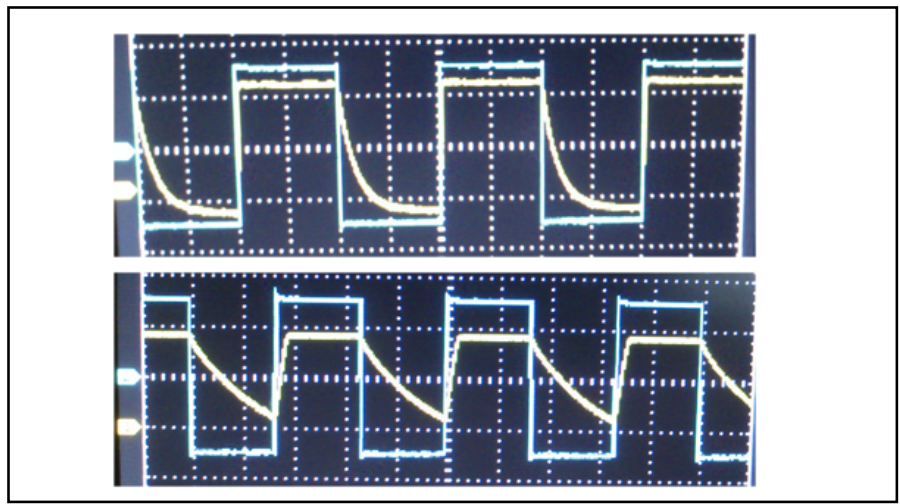

Fig.1. Waveforms of LED as Sensor

In Fig. 1 two waveforms received by LED is shown, which are $500 \mathrm{~Hz}$ and $3 \mathrm{KHz}$ respectively. We have noted that quality of waveform degraded at higher frequency. We can go further this frequency too but we have to use filter and amplifier as far as communication is concern. The waveforms are for indication purpose only, because it depends on length between transmitter and receiver, external lighting condition, and noise. We can't ignore the fact that LED is not coherent light source like LASER is. So long distance can't be achieved. But we can use reflector at transmitter side to focus light and magnifying optics at receiver side to concentrate light [8].

\section{VLC FOR MOBILE HANDSETS}

Today all mobile phones uses flash for camera or as emergency light for night. There is not any additional use of it. In LED white light is produced by blue emission which excite yellow phosphor layer on top of the LED. So we can say white light is generated by combining Blue and Yellow radiation. 
In suggested scheme we can use Mobile Handset flash to transmit data. Researchers have already achieved higher data rate with White LEDs[9] .But as we know that white light can be generated by Red, Green, Blue lights too, which is what we are interested in. In proposed method we suggest to use this 3 color light to produce white light for future mobile applications. In mean while someone can use these LEDs as sensors to receive data too when flash is not being used. As shown in Fig. 2 we have used RGB led which was producing White Light (this is the fact everybody know already, but included for better understanding of readers).

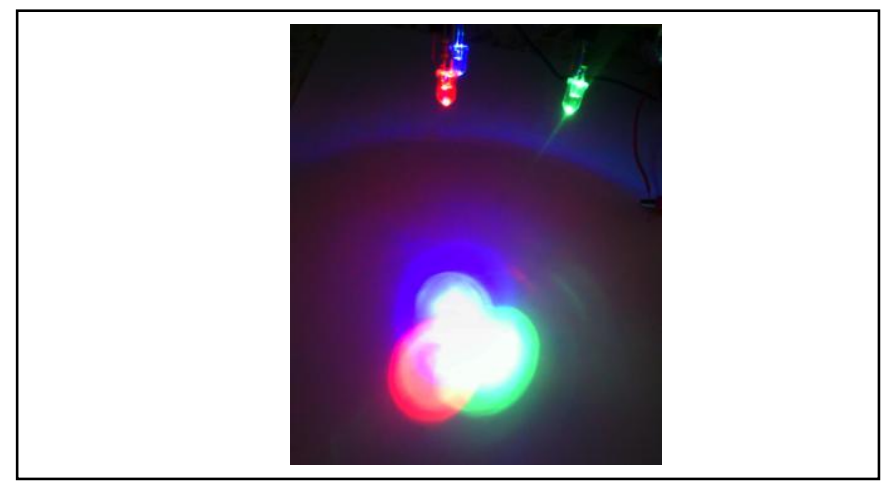

Fig.2. R-G-B LEDs to produce white light.

We can use this technique in mobile handsets in which LEDs are to be connected to microcontroller of the mobile to receive data as well. As most of the mobile handsets don't use flash all the time we can use this for low level data communication like to exchange text or midi tones. But it is also the fact that Blue LED is not good as far as sensing is concerned. And Green can't receive light from Red and Orange LEDs. But Red LED is better receiver. So we can use Red LED to receive data. And other LED to produce white light when data is not being received.

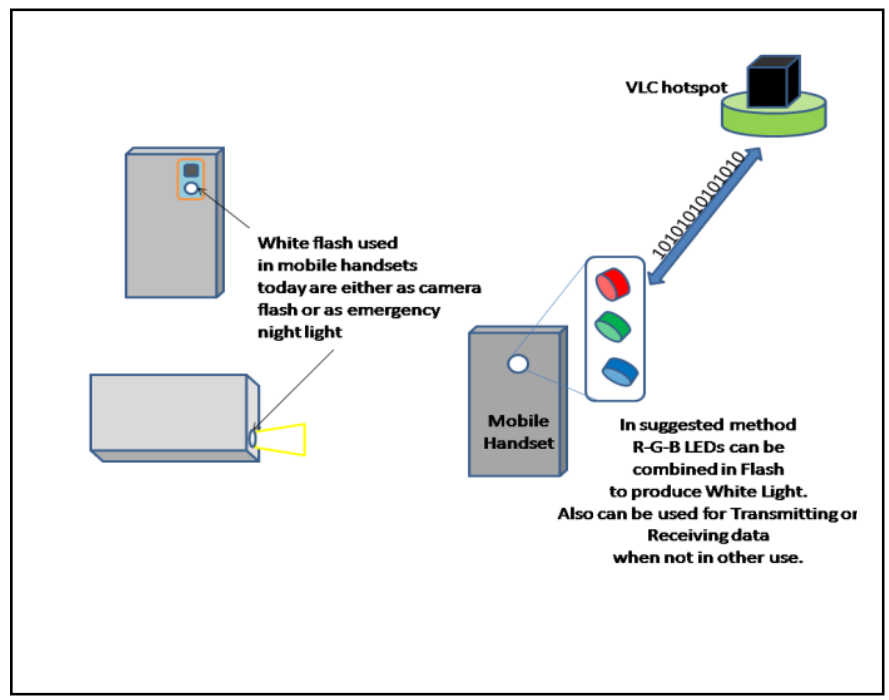

Fig.3. Suggested model for flash in Mobile Handsets.

We can see in Fig.3 where we have shown such model, which can be implemented with this technology. Performance of LED can be improved with filter and amplifiers. But there is also a limit. More and more manufacturer should do research and development to produce such hybrid LEDs which can be used for bi-directional communication. We have used $5 \mathrm{~mm}$ clear lens LED but one can also try different LED to get improved result along with good photo diode amplifier.

We should also note that all the home appliances we use today have LED as power or other function indicator. So we can implement this technology there as well. Applications are endless only limited by the imagination of someone.

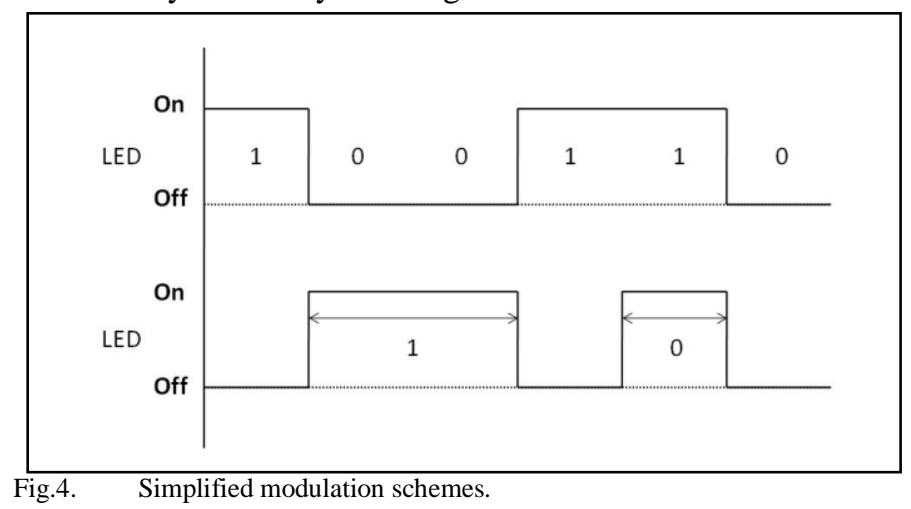

Modulation scheme is also important in this regard. We can do parallel communication here so appropriate scheme should be selected according to our application. OOK, PWM, OFDM, etc are the popular among researchers for visible light communication [10]. We can even build our low level protocol to develop applications. This is what we are doing our research on.

\section{RESULTS}

As we noted earlier that some LED can "sense" only some LED lights, in our experiments we had two set up where in first set-up two microcontrollers used as Transmitter and Receiver. From transmitter we have 4 switches which are supposed to control 4 equipment (LED in our case).the transmission medium was Red LED as transmitter and another Red LED as receiver. At the same time in second set up we have used Blue LED as transmitter connected with function generator with certain frequency and Green LED as receiver. Distance between both transmitter and receiver was $\sim 3 \mathrm{~cm}$.both $\mathrm{Tx}$ and $\mathrm{Rx}$ worked individually without any loss even placed side by side in such a way that both transmitters have $1 \mathrm{~cm}$ distance only and same was done on receiver side. We should note here that when we decreased distance so lower to place them very near at that time Red LED was unable to sense switching command send by the transmitter Red LED due to the interference of Blue and Green LEDs. But with approx $3 \mathrm{~cm}$ distance we were able to switch on LED/devices at receiver side (with Red LED to Red LED) at the same time we were able to receive waveforms at second receiver (with Blue LED to Green LED).we selected Red LED for first configuration intentionally as we wanted to try same color for transmission and reception purpose.

All experiments are in primary stage as one has to make this technology more effective and resolve noise problem. Here choosing modulation scheme is also very important. There might be many use of this technology but in current time we 
use camera flash not even for data transmission purpose. We encourage more and more research to be done for this.

Here LED was working with such low frequency so we can do communication with two methods shown in Fig .4. Where first is a scheme in which we can set timer for both side and can send data with changing the state of LED on or off. Popularly On-Off keying. Synchronization is most important unless we will be compromising with data rate. In second scheme we don't have to set timer as we defining certain time for both 1 and 0.therefore if LED will successfully sense light for defined time it will be easy for us to consider it as 1 or 0.popularly known as Morse code where dot-dash used in similar way. This is simple scheme which can be used for development purpose.

\section{FUTURE WORK}

Data rates are matter of concern here so work should be done to achieve better data rates by using different LEDs, better /high end hardware like FPGAs, or appropriate modulation scheme.

In our experiments we were limited by some $\mathrm{cm}$ distance because we didn't use reflector and magnifying optics and not even amplifier/filter. One can achieve better light gathering by this like astronomy telescope do.

\section{CONCLUSION}

In this paper we have shown how VLC is useful over Radio Frequencies at some places. We have also shown that different color LED can sense different lights. According to our application we can select different color LEDs. In suggested model we have shown idea of improved flash with WDM which can be used as data transceiver. We are also continuing research to implement this technology successfully and finding other ways to improve results. Other applications can be done also using this technology, and list of it will never end.

\section{REFERENCES}

[1] H. Le Minh , D. O. ;Brien , G. Faulkner , L. B. Zeng, K. Lee , D. Jung , Y. Oh and E. T. Won "100-Mb/s NRZ visible light communications using a post equalized white LED", IEEE Photon. Technol. Lett., vol. 21, no. 15, pp.1063 -1065 2009.

[2] Elgala, H., Mesleh, R., Haas, H., Pricope, B.," OFDM Visible Light Wireless Communication Based on White LEDs", IEEE 65th Vehicular Technology Conference, 2007. VTC2007-Spring., 22-25 April 2007, 2185 - 2189.

[3] Forrest M. Mims III," Siliconnections: Coming of Age in the Electronic Era", McGraw-Hill, New York, NY, 1986.

[4] Forrest M. Mims III, "LED Circuits and Projects", Howard W. Sams and Co., Inc., New York, NY, pp. 60-61, 76-77, 122-12.

[5] Khan, T.A., Tahir, M., Usman, A., "Visible light communication using wavelength division multiplexing for smart spaces", Consumer Communications and Networking Conference (CCNC), 2012 IEEE,1417 Jan, $230-234$.

[6] P. Dietz, W. Yerazunis, and D. Leigh, "Very low-cost sensing and communication using bidirectional leds," in UbiComp 2003: Ubiquitous Computing. Springer, 2003, pp. 175-191.

[7] S. Schmid, G. Corbellini, S. Mangold, T. R. Gross, "An LED-to-LED Visible Light Communication System with Software-Based Synchronization", GLOBECOM Workshop (OWC), 3-7 Dec, 2012, Anaheim, CA, USA

[8] DJ Varanva, KMVV Prasad, "Various Aspects of Visible Light Communication and its applications", e-DCSECT-2013, International Journal of Electronics and Communication Technology (IJECT) Volume 4, Spl - 2 / Jan - March 2013,105-107.

[9] A. M. Khalid, G. Cossu, R. Corsini, P. Choudhury, E. Ciaramella," 1$\mathrm{Gb} / \mathrm{s}$ Transmission Over a Phosphorescent White LED by Using Rate Adaptive Discrete Multitone Modulation", Volume 4,Number 5, October 2012,1465-1473.

[10] O'Brien, D. C. Zeng, L., Le-Minh, H., Faulkner, G., Walewski, J.W., Randel S.,"Visible Light Communications: challenges and possibilities", Personal, Indoor and Mobile Radio Communications, 2008. PIMRC 2008. IEEE 19th International Symposium, 15-18 Sept. 2008, 1-5. 